\title{
The Applications of an Interdiffused Quantum Well in a Normally On Electroabsorptive Fabry-Perot Reflection Modulator
}

\author{
Wallace C. H. Choy, Student Member, IEEE, and E. Herbert Li, Senior Member, IEEE
}

\begin{abstract}
A Fabry-Perot reflection-type modulator which uses interdiffused AlGaAs/GaAs quantum wells as the active cavity material has been studied and optimized theoretically. An asymmetric Bragg reflector structure (modeled by transfer matrices), with a doped depletion layer in the heterostructure, has been considered. This is the first study to model such a material system in this type of modulator, and the results show improvement in modulation property over its as-grown rectangular quantumwell modulator. In particular, the change of reflectance in the diffused quantum-well modulator is almost 0.6 to 0.7 , which is higher than that of the typically available values $(\sim 0.5$ to 0.6), while the OFF-state on-resonance reflectance is almost close to zero. The operation voltage is also reduced by more than half as the interdiffusion becomes extensive. The finesse of the more extensively diffused quantum well also increases. Both of these features contribute to an improvement of the change of reflectance in the modulator. The operation wavelengths can be adjusted over a range of $100 \mathrm{~nm}$. However, the absorption coefficient change of the diffused quantum well increases only when there is a small amount of interdiffusion.
\end{abstract}

\section{INTRODUCTION}

$\mathbf{I}$ N RECENT YEARS, there has been an increasing interest in high-performance Fabry-Perot (FP) reflection modulators. It is very common to use quantum-well (QW) materials with electroabsorptive and electrooptic properties in the FP cavity [1]-[4]. In order to improve the performance of the modulator, various QW [5], [6] and superlattice [7] structures have been employed in the active region of the FP cavity. Recently, the diffused quantum-well (DFQW) materials and their applications have been considered in some detail [8] for applications in optoelectronic devices. However, no detailed study on the modulation properties of active DFQW materials in a FP reflection modulator has been reported. To our knowledge, this is the first study to model such material in this type of modulator. In this paper, a $101-\AA / 100$ $\AA \mathrm{Al}_{0.3} \mathrm{Ga}_{0.7} \mathrm{As}-\mathrm{GaAs}$ DFQW structure is employed as the active layer in a FP refection modulator.

There still remains a challenge to both symmetric and asymmetric FP structures in terms of precise control of the active QW layer thickness and composition. Due to this reason, it becomes difficult to operate the modulator precisely at

Manuscript received January 2, 1996; revised September 9, 1996. This work was supported by the University of Hong Kong CRCG. The work of W. C. H. Choy was supported by the Sir Edward Youde Memorial Fellowship.

The authors are with the Department of Electrical and Electronic Engineering, University of Hong Kong, Pokfulam Road, Hong Kong.

Publisher Item Identifier S 0018-9197(97)01565-0. the desired wavelength with good modulator performance. Several ways to modify the operation wavelength, $\lambda_{\text {op }}$, either permanently or nonpermanently have been reported [2], [9], [10]. In this paper, we will introduce the new method of using interdiffusion technology to adjust the operation wavelength. The conventional permanent method, which only applies to the reflection modulators, is to incorporate a transmission modulator and a corner-cube retroreflector. This provides a means to finely adjust the $\lambda_{\mathrm{op}}$ to match the source [11]. Alternatively, the $\lambda_{\text {op }}$ may be modified nonpermanently through the application of an electric field to the FP modulator. This provides a quantum-confined Stark effect (QCSE) for QW and Wannier-Stark localization for a superlattice [9], [10], [12]. It should be noted that apart from its ability to adjust $\lambda_{\mathrm{op}}$ in the QW cavity structure, the QCSE can also be employed to modify the reflectance at an operation wavelength for the FP modulator. Although most rectangular QW's and regular superlattice have been optimized for the FP reflection modulators, still very few nonrectangular wells have been optimized for such modulators. In fact, to our knowledge, there is only one report demonstrating the disordered GaAs-AlGaAs QW FP modulator [13]. No investigation has yet been made so far to determine the optimum performance of the DFQW FP modulator. It is also not cost-effective to optimize the reflection modulation through costly experiments. This is the main reason why we are developing a computational model for this modulator.

A thermally interdiffused QW structure is proposed here as an intrinsic material for the active region of the FP cavity. This permits $\lambda_{\text {op }}$ and the optical properties of the modulator to be finely adjusted in a controllable manner by annealing the as-grown QW. It works as follows. Interdiffusion of the QW structure will increase the transition energy (and thus increases $\lambda_{\text {op }}$ ) from both heavy and light holes in the valence band to electron states in the conduction band and modify the confinement of these carriers in the QW. As a consequence, the joint density of states changes and the optical parameters of the QW material vary. This modification of $\lambda_{\mathrm{op}}$ in the FP modulator provides an alternative option apart from the permanent and the nonpermanent method mentioned above. Details on the theories and experiments of the DFQW can be found in [14]-[18], respectively. In fact, the technology of DFQW has been employed in the development of transistors [19], modulators [13], lasers [20], waveguides [21], and the integration of the latter two [22]. These applications show 
that the DFQW structure not only provides an adjustability in $\lambda_{\mathrm{op}}$ but also a modification of the device's operation performance. This further consolidates the capability of using the interdiffusion technology in developing optoelectronic components and its integration technology.

The aim of this paper is to study the effects of the modified optical properties of $\mathrm{Al}_{0.3} \mathrm{Ga}_{0.7} \mathrm{As}-\mathrm{GaAs} \mathrm{DFQW}$ and the QCSE of different applied fields' strength on the vertical asymmetric FP reflection modulator. In Section II, we present the modeling of QW optical parameters in a stacked FP modulator structure including the built-in and external potentials. The relation between reflectance change and the change of absorption coefficient, as well as between the change of refractive index and finesse, are also included to facilitate a more comprehensive study on the contribution of DFQW to modulation. In Section III, we present and discuss intuitively the important properties of the DFQW material system for a FP modulator, followed by its operation criteria. This includes the investigation of the total change of reflectance and the relative reflectance change, as well as qualitatively study of the characteristics and performances of the DFQW structure in the FP modulator. Finally, in Section IV, conclusions will be drawn.

\section{MODELING}

Fig. 1 is a flowchart showing how the performance of a DFQW FP reflection modulator is calculated. The mainflow can simply be explained as follows. The upper half of the flowchart mainly involves the calculations of the optical parameters, the real part of the total dielectric function $\varepsilon_{1}^{T}$ and imaginary parts of the dielectric function for $\Gamma$ valley $\varepsilon_{2}^{\Gamma}$, the total absorption coefficient $\alpha^{T}$ and the real part of refractive index $n_{r}$, of the DFQW structure. These parameters will be used to simulate the whole device structure. In order to determine the reflectance $\left(R_{\text {tot }}\right)$ of the modulator [see lower half of the flowchart (Fig. 1)], transfer matrices method will be employed and will be discussed in Section II-C). This is a standard method to express the relation between the incidental optical electric field (both forward and backward directions of propagation) and the output field passing the mirror structure. By careful selection of the $\lambda_{\mathrm{op}}$ 's and adopting optimized materials for the QW and Bragg reflectors, the $R_{\mathrm{FP}}$ can be determined. These include the thickness of alternative layers of the FP mirror and the DFQW structure, and the number of these layers. In Section II-D, the thickness in the depletion region of the layered structure with different dielectric constants is calculated in order to determine the applied voltage of the devices. Finally, in Section II-E, some relations are developed to interpret the effects of modified optical parameters caused by interdiffusion on the performance of the FP modulator.

\section{A. FP Device Structure}

The schematical device structure is shown in Fig. 2 where the intrinsic layer consists of 61 periods of $101-\AA \AA / 100-\AA$ $\mathrm{Al}_{0.3} \mathrm{Ga}_{0.7} \mathrm{As}-\mathrm{GaAs}$ DFQW's. This DFQW structure is sandwiched between two p- and n-type doped quarter-wavelength

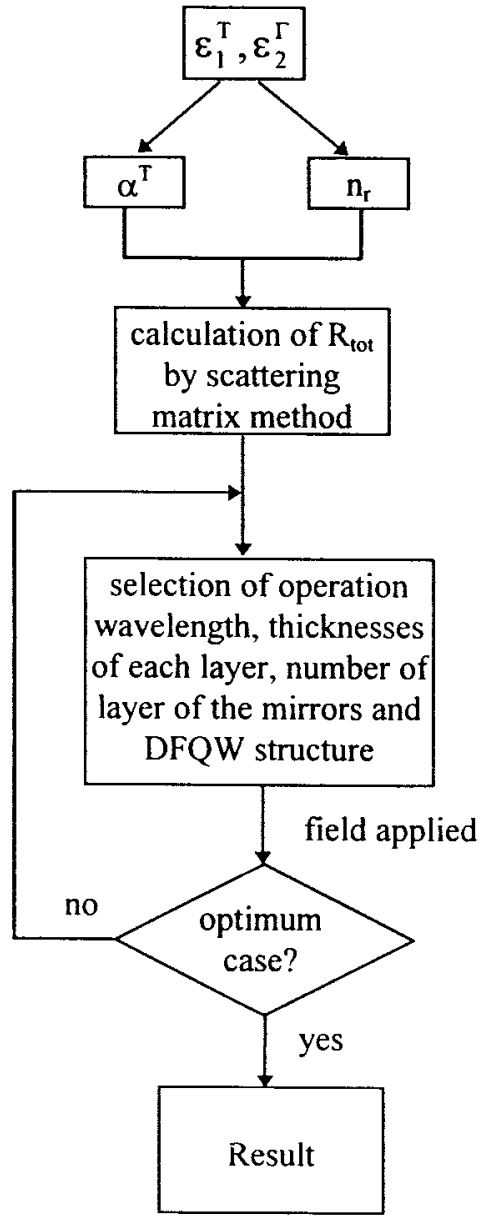

Fig. 1. The flowchart to model and develop a high-performance reflection modulator.

Bragg reflectors. The Bragg reflector consists of periods of alternative $\mathrm{Al}_{0.3} \mathrm{Ga}_{0.7} \mathrm{As}$ and $\mathrm{AlAs}$ layers. The whole structure will grow on a $\mathrm{n}^{+}-\mathrm{GaAs}$ substrate, assuming it to be coated by a thin layer of $\mathrm{Au}$ on both the top and bottom as electrical contacts. This structure serves as an asymmetric normally ON electroabsorptive FP modulator operating at the biased exciton peak. It should be noted that any change of absorption will be followed by the change of refractive index. It can be clearly explained from the Kramers-Krönig transformation between $\Delta \alpha$ and $\Delta n$ under the sum rules [23], as shown below:

$$
\Delta n(\omega)=\frac{c}{\pi} \mathrm{PV} \int_{\omega_{1}}^{\omega_{2}} \frac{\Delta \alpha\left(\omega^{\prime}\right) d \omega^{\prime}}{\left(\omega^{\prime}\right)^{2}-\omega^{2}}
$$

where PV stands for the principle value and $\Delta \alpha$ of the DFQW is considered within the corresponding spectral interval $\left(\omega_{1}, \omega_{2}\right)$. The $\Delta n$ will cause shifting of the FP mode in the modulator. In modeling the DFQW device structure here, the effect of both $\Delta n$ and $\Delta \alpha$ are taken into consideration.

\section{B. Active DFQW Materials-Optical Parameters}

Assuming that all barriers are thick enough to provide good quantum confinement in the wells, the interdiffusion of the DFQW structures is studied theoretically using an error function profile [24]-[28]. The extent of interdiffusion can be measured by a diffusion length $L_{d}$, defined as $L_{d}=(D t)^{1 / 2}$, 


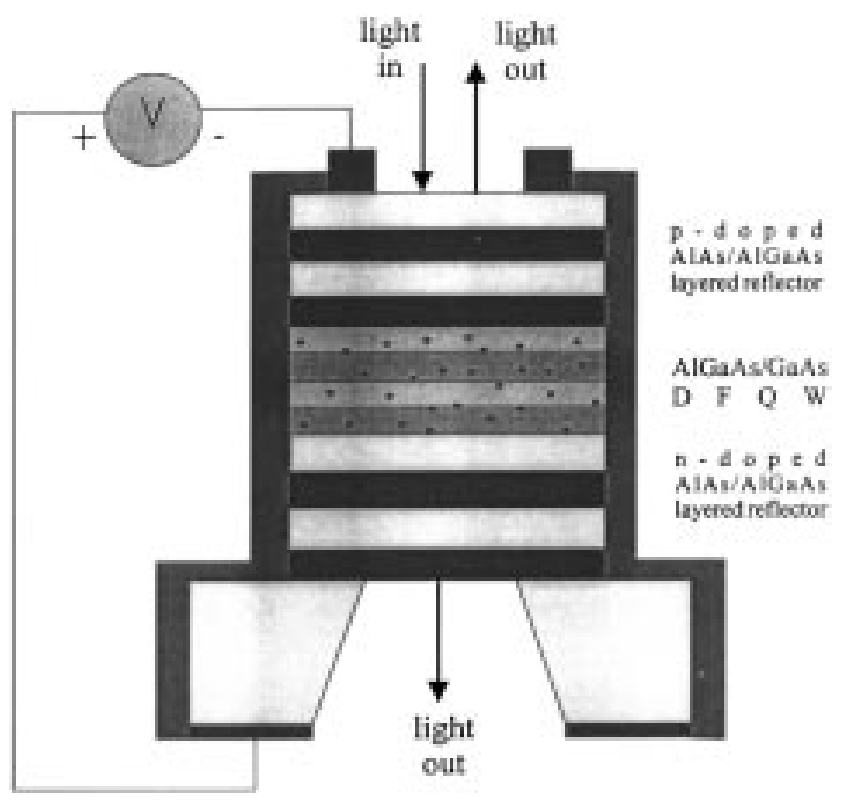

(a)

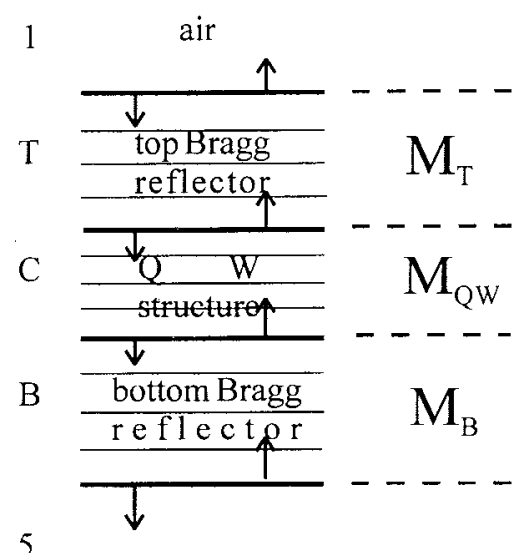

(b)

Fig. 2. Schematic of the layer sequence of the FP reflection layer. The active cavity is 61 periods $\mathrm{Al}_{0.3} \mathrm{Ga}_{0.7} \mathrm{As}-\mathrm{GaAs}$ intrinsic interdiffused MQW structure with a periodic thickness $101-\AA / 100-\AA$, respectively. The structure is sandwiched with two reflectors, produced by two alternative layers $\mathrm{Al}_{0.3} \mathrm{Ga}_{0.7} \mathrm{As}$ and $\mathrm{AlAs}$ the top is p-doped while the another one growth on $\mathrm{n}^{+}-\mathrm{GaAs}$ is $\mathrm{n}$-doped by ion implantation.

where $D$ is the material-dependent diffusion coefficient and $t$ is the annealing time. The stage of interdiffusion starts with a small $L_{d}$ where QW compositional profile remains almost rectangular with a graded interface. As $L_{d}$ increases further, the interdiffusion of wells becomes extensive, which results in complete interdiffusion, as $L_{d}$ approaches infinity an averaged bulk material between the well and barriers results.

The imaginary part of the dielectric function for the $\Gamma$ valley, $\varepsilon_{2}^{\Gamma}$, is obtained by

$$
\varepsilon_{2}^{\Gamma}(\omega)=\varepsilon_{2}^{\text {exc }}(\omega)+\varepsilon_{2}^{\text {bound }}(\omega)+\varepsilon_{2}^{\text {con }}(\omega)
$$

where $\omega$ is the angular frequency, $\varepsilon_{2}^{\text {exc }}$ is the $1 \mathrm{~S}$ exciton contribution derived by the density-matrix approach at the subband edge without the influence of band mixing, and $\varepsilon_{2}^{\text {bound }}$ is conduction-valence band bound-state contribution without the electron-hole interaction. $\varepsilon_{2}^{\text {con }}$ is the contribution from the unbound continuum states above the barrier. Details of these calculations are given in [17]. The real part of the dielectric function is $\varepsilon_{1}^{T}$, given by

$$
\varepsilon_{1}^{T}(\omega)=\varepsilon_{1}^{\Gamma}(\omega)+\varepsilon_{1}^{X, L}(\omega)
$$

where $\varepsilon_{1}^{\Gamma}$ and $\varepsilon_{1}^{X, L}$ are the real parts of the dielectric function for the $\Gamma$ valley and $X$ and $L$ regions, respectively. They are determined by using the Kramers-Krönig transformation of the imaginary part of the dielectric function for $\Gamma, X$, and $L$ valley, respectively [29]. The $n_{r}$ is now given by [30]

$$
n_{r}(\omega)=\left({ }^{1} / 2 \varepsilon_{1}^{T}(\omega)+1 / 2\left\{\left[\varepsilon_{1}^{T}(\omega)\right]^{2}+\left[\varepsilon_{2}^{\Gamma}(\omega)\right]^{2}\right\}^{2}\right)^{1 / 2} .
$$

The unpolarized absorption coefficient $\alpha^{T}(\omega)$ is the sum of $\alpha^{\text {bound }}(\omega), \alpha^{\operatorname{exc}}(\omega)$, and $\alpha^{\operatorname{con}}(\omega)$. They are the absorption coefficient of the bound state, the exciton state, and the continuum region, respectively. The different parts of the absorption coefficient can be calculated from the corresponding parts of the dielectric functions in $\varepsilon_{2}^{\Gamma}(\omega)$ using the relation in [30]. $\alpha^{\gamma}(\omega)=\left[\omega \varepsilon_{2}^{\gamma}(\omega)\right] /\left[c_{0} n_{r}(\omega)\right]$, where the superscript $\gamma$ signifies either the bound state, exciton state, or the continuum region. $c_{0}$ and $n_{r}(\omega)$ are the speed of light in vacuum and the refractive index of the DFQW, respectively.

\section{FP Device-Reflectors and Cavity}

The modeling of the device using transfer matrices are divided into two main parts: 1) mirrors (top and bottom) and 2) intrinsic DFQW cavity. The mirrors are used to serve as reflectors and control the light interaction within the DFQW material. The DFQW material, on the other hand, is used to modulate the light by modifying its $\Delta n$ and $\Delta \alpha$.

The top and bottom mirror are made up of alternative layers, i.e., layers of low $(L)$ and high $(H)$ refractive index, in order to obtain an optimized reflectance; each of these layers has to keep a phase change of $\pi / 2$ (quarter wave) at the OFF state $\lambda_{\text {op }}$ [31]. The thickness of each layer of the Bragg reflector can be increased to include an extra multiple of $\pi$ phase change as compared to the original quarter-wave case. This increases the number of FP modes, and thus the modulator can choose to operate at a larger number of FP modes. However, the drawback is an enlargement of the total thickness of the device. In addition, not all FP modes can operate under high $\Delta R_{\text {tot }}$ and high $\Delta R_{\text {tot }} / R_{\text {tot }}(\min )$ modulation conditions. The full transfer matrices at the top reflector of our device (without any residual loss) are shown in (5), shown at the bottom of the next page, where $r_{i j}\left(t_{i j}\right)$ is the refractive (transmission) coefficient when the field propagates from layer $i$ to $j$ such that $r_{i j}=\frac{n_{i}-n_{j}}{n_{i}+n_{j}}, t_{i j}=\frac{2 n_{i}}{n_{i}+n_{j}}, n_{i}$ and $n_{j}$ are the refractive indexes of medium $i$ and $j$, respectively (each square bracket represents the transfer matrix of one layer). The subscript $a, L$, $H$, and $c$ stand for air, AlAs (low refractive index), AlGaAs (high refractive index), and the DFQW cavity, respectively. The exponential imaginary value $p_{i}=\sqrt{-1}\left(\delta_{i} l_{i}\right), \delta_{i}=$ $2 \pi n_{i} / \lambda$, where $l_{i}$ is the thickness of layer $i$ and $\lambda$ is the photon wavelength and $\sqrt{-1}$ indicates an imaginary value. The bottom matrix $\left(M_{B}\right)$ can be expressed in a similar way to that of the top $\left(M_{T}\right)$. The main differences lie in the upper and lower adjacent medium of the two reflectors, where the upper 
adjacent medium of the bottom reflector should be DFQW instead of air and the lower medium of that reflector should be GaAs substrate instead of DFQW for the top reflectors. Together with the cavity structure, the transfer matrices of the whole structure $M_{\mathrm{FP}}$ can be written as

$$
\begin{aligned}
\left(M_{\mathrm{FP}}\right)= & \left(M_{T}\right) \\
& \times\left(\begin{array}{cc}
e^{\frac{1}{2} \alpha_{c} l_{c}-\sqrt{-1}\left(\delta_{c} l_{c}\right)} & 0 \\
0 & e^{-\frac{1}{2} \alpha_{c} l_{c}+\sqrt{-1}\left(\delta_{c} l_{c}\right)}
\end{array}\right)\left(M_{B}\right)
\end{aligned}
$$

where the phase change per unit length (of cavity) is defined as $\delta_{c}=2 \pi n_{c} / \lambda$, when the effect of penetration depth has been neglected, $\alpha_{c}$ is the absorption coefficient for the DFQW cavity, $l_{c}$ is the cavity length, and the terms $\alpha_{c} l_{c} / 2$ and $\sqrt{-1}\left(\delta_{c} l_{c}\right)$ indicate the residual loss and phase change of the field in the DFQW medium after traveling a half trip, respectively. The reflectance $R_{\text {tot }}$ of the entire FP modulator can now be given by

$$
R_{\mathrm{tot}}=\left|\frac{\sqrt{R_{T}}-\sqrt{R_{B}} e^{-\alpha_{c} l_{c}+2 \sqrt{-1}\left(\delta_{c} l_{c}\right)}}{1-\sqrt{R_{T}} \sqrt{R_{B}} e^{-\alpha_{c} l_{c}+2 \sqrt{-1}\left(\delta_{c} l_{c}\right)}}\right|^{2}
$$

where $R_{T}\left(R_{B}\right)$ is the reflectance of the top (bottom) mirror. It should be noted that a quarter-wavelength Bragg reflector structure, $L(H L)^{N}$, is constructed ( $N$ is the period of alternative layers). The $L(H L)^{N}$ denotes the arrangement of the low $(L)$ and high $(H)$ refractive-index alternative layer structure. In principal, a theoretical infinite $\Delta R_{\text {tot }} / R_{\text {tot }}(\min )$ ratio can be achieved when the impedance-matching condition holds, i.e., $R_{T}-R_{B} \exp \left(-2 \alpha_{c} l_{c}\right)=0$. The modulated amplitude of the optical field is obtained under this condition, which is only valid when the cavity phase-matching condition has been satisfied. This implies phase change in the cavity is a multiple of $2 \pi$ after traveling a round trip [5], [7], [32]. Finally, by choosing a value for $R_{B}, R_{T}$ can be estimated.

\section{D. $p$-i-n Device-Field-Induced Depletion Region}

In this section, the built-in voltage and applied voltage for operation are determined. Assuming that the materials are homogenous in the direction perpendicular to the grown axis of the device, a one-dimensional (1-D) approach is considered. The thickness of the depletion region, $X_{m}$, in the alternative layered structure with different dielectric constants is calculated using Poisson's equation with appropriate boundary conditions. The electrostatic potential $V$ inside the depletion region can be expressed as

$$
V=V(\text { build-in })-V(\text { applied })=\Psi\left(-X_{n}\right)-\Psi\left(X_{p}\right)
$$

where $\Psi\left(-X_{n}\right)$ and $\Psi\left(X_{p}\right)$ are the potentials at $-X_{n}$ and $X_{p}$, respectively, which are the coordinates (zero reference taken at the i-p interface) of the depleted end points in the ndoped and p-doped reflectors, respectively. The $\Psi\left(-X_{n}\right)$ and $\Psi\left(X_{p}\right)$ considered in the Poisson's equation are expressed in terms of the depletion thickness and the carrier density of the multilayered materials in the stacked structure. The integrated form of the Poisson's equation is expressed as a quadratic equation for the depletion layer $X_{m}$ as shown below:

$$
\begin{gathered}
\left(\frac{N_{D}}{2 \varepsilon_{i}^{T}} a^{2}+\frac{N_{A}}{2 \varepsilon_{j}^{B}} g^{2}\right)^{2} X_{m}^{2}+\left(\frac{N_{D}}{\varepsilon_{i}^{T}} d a-\frac{N_{A}}{\varepsilon_{j}^{B}} d g-\zeta\right) X_{m} \\
+\left[\frac{N_{D}}{2 \varepsilon_{i}^{T}}+\frac{N_{A}}{2 \varepsilon_{j}^{B}}\right]\left(b_{j} l_{c}\right)^{2}+\Xi-\frac{V}{q}=0
\end{gathered}
$$

where

$$
\begin{aligned}
a= & \frac{N_{A}}{N_{A}+N_{D}}, \quad d=\left(\frac{N_{D}-N_{c}}{N_{d}+N_{A}}\right)\left(l_{c}\right) \\
c= & \frac{N_{D}}{N_{A}+N_{D}}, \quad g=\frac{N_{D}}{N_{D}+N_{A}} \\
\zeta= & N_{A} S_{2}^{B}+N_{D} S_{2}^{T}+l_{c}\left(\frac{N_{d} a}{\varepsilon_{1}^{T}}-\frac{N_{A} c}{\varepsilon_{c}}\right) \\
\Xi= & N_{D}\left(\frac{S_{1}^{T}}{2}-S_{2}^{T} d\right)+N_{A}\left(\frac{S_{1}^{B}}{2}+S_{2}^{B} d\right) \\
& +\frac{\left(l_{c}\right)^{2}}{2}\left[\frac{N_{D}}{\varepsilon_{1}^{T}}-\frac{N_{c}}{\varepsilon_{c}}\right]-d l_{c}\left[\frac{q N_{D}}{\varepsilon_{1}^{T}}+\frac{q N_{A}}{\varepsilon_{c}}\right] \\
S_{1}^{T}= & \sum_{i=2}^{Y}\left[\frac{1}{\varepsilon_{i}^{T}}-\frac{1}{\varepsilon_{i-1}^{T}}\right]\left(t_{i-1}\right)^{2} \\
S_{2}^{T}= & \sum_{i=2}^{Y}\left[-\frac{1}{\varepsilon_{i-1}^{T}}+\frac{1}{\varepsilon_{i}^{T}}\right]\left(t_{i-1}\right) \\
S_{1}^{B}= & \sum_{j=2}^{Z}\left[\frac{1}{\varepsilon_{j}^{B}}-\frac{1}{\varepsilon_{j-1}^{B}}\right]\left(b_{j-1}\right)^{2} \\
S_{2}^{B}= & \sum_{j=2}^{Z}\left[\frac{1}{\varepsilon_{j}^{B}}-\frac{1}{\varepsilon_{j-1}^{B}}\right]\left(b_{j-1}\right) .
\end{aligned}
$$

$\varepsilon_{i}^{T}$ and $\varepsilon_{j}^{B}$ are the $x$-independent terms of the dielectric constant in the $i$ layer and $j$ layer of the top and bottom reflector, respectively. $N_{A}, N_{c}$, and $N_{D}$ are the doping concentrations of the $\mathrm{p}-\mathrm{i}-\mathrm{n}$ structure with values around orders

$$
\begin{aligned}
& M_{T}=\left(\begin{array}{cc}
1 / t_{a L} & 0 \\
\frac{r_{a L}}{t_{a L}} & -1
\end{array}\right)\left[\left(\begin{array}{cc}
1 & r_{a L} \\
0 & -t_{L a}
\end{array}\right)\left(\begin{array}{cc}
e^{-P_{L}} & 0 \\
0 & e^{P_{L}}
\end{array}\right)\left(\begin{array}{cc}
1 / t_{L H} & 0 \\
r_{L H} / t_{L H} & -1
\end{array}\right)\right]
\end{aligned}
$$

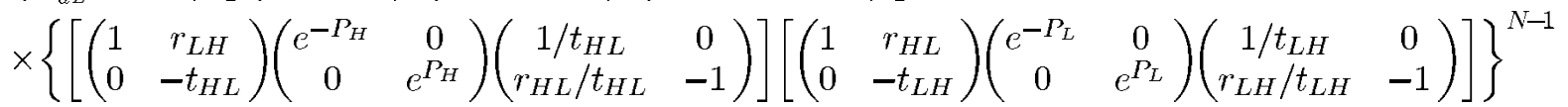

$$
\begin{aligned}
& \times\left[\left(\begin{array}{cc}
1 & r_{L H} \\
0 & -t_{H L}
\end{array}\right)\left(\begin{array}{cc}
e^{-P_{H}} & 0 \\
0 & e^{P_{H}}
\end{array}\right)\left(\begin{array}{cc}
1 / t_{H L} & 0 \\
r_{H L .} / t_{H L} & -1
\end{array}\right)\right]\left[\left(\begin{array}{cc}
1 & r_{H L} \\
0 & -t_{L H}
\end{array}\right)\left(\begin{array}{cc}
e^{-P_{L}} & 0 \\
0 & e^{P_{L}}
\end{array}\right)\left(\begin{array}{cc}
1 / t_{L c} & 0 \\
r_{L c} / t_{L c} & -1
\end{array}\right)\right]\left(\begin{array}{cc}
1 & r_{L c} \\
0 & -t_{c L}
\end{array}\right)
\end{aligned}
$$


of $10^{18}-10^{14}-10^{18} \mathrm{~cm}^{-3}$, respectively, and $l_{c}$ is the thickness of the DFQW structure, $q$ is carrier charge, $t_{i}$ and $b_{j}$ are the depletion width extensions of the layer $i$ (top reflector) and layer $j$ (bottom reflector), respectively, due to the applied field. $i=Y$ and $j=Z$ are the outermost layers of the top and bottom reflectors to which the depletion range will extend. The applied voltage which is equivalent to the expected electric field in the depletion layer can then be calculated. Therefore, the voltage swing of the modulator can be determined. However, the QCSE induced in the DFQW cavity is a consequence of the applied electric field (not of voltage swing). Therefore, in the forecoming sections, the potential $(\mathrm{kV} / \mathrm{cm})$ mentioned will be the applied electric field instead of the voltage swing.

\section{E. Effects of Optical Properties of the Active Region in the FP Modulator}

Since interdiffusion will modify the QW structure by intermixing the barrier constituents with that of the well, this affects the optical properties (absorption coefficient and refractive index) in the DFQW cavity. As a consequence, $\Delta R_{\text {tot }}$ is modified from the case of the rectangular QW. In fact, $\Delta \alpha_{c}$ (due to applied field) modifies the value of on-resonance reflectance while $\Delta n_{c}$ creates shifting in the operation wavelength of the FP mode. We make the following assumptions:

1) $\Delta \lambda_{\mathrm{op}}=0$ implies ignoring the shift of the FP mode due to $\Delta n_{c}$;

2) $\mathrm{OFF}$ state $R_{\text {tot }}=0$ implies that the FP modulator can operate at an OFF-state on-resonance mode perfectly while holding the impedance and phase-matching conditions;

3) $R_{T}$ and $R_{B}$ are both $<1$ and remain constant under bias, which is a reasonable assumption since the $\lambda_{\text {op }}$ used is far from the bandgap of the bulk materials of the alternative layers in the reflectors, and hence, the change of their refractive index can be neglected.

Under these assumptions, the relation from (7) between $\Delta \alpha_{c}$ and $\Delta R_{\text {tot }}$ can then be expressed as

$$
\Delta R_{\mathrm{tot}}=\left|\frac{\sqrt{R_{T}}-\sqrt{R_{T}} e^{\Delta \alpha_{c} l_{c}}}{1-R_{T} e^{\Delta \alpha_{c} l_{c}}}\right|^{2}
$$

where $\Delta \alpha_{c}=\alpha_{c}(\mathrm{OFF})-\alpha_{c}(\mathrm{ON})$ and which is greater than zero in our normally ON DFQW modulator. This implies $\exp \left(\Delta \alpha_{c} l_{c}\right)$ is greater than 1 . With this condition and by taking derivative with respect to $\exp \left(\Delta \alpha_{c} l_{c}\right),(10)$ becomes

$$
\frac{d\left(\Delta R_{\text {tot }}\right)}{d\left(\exp \left(\Delta \alpha_{c} l_{c}\right)\right)}= \pm \frac{\sqrt{R_{T}}\left(1-R_{T}\right)}{\left(1-R_{T} \exp \left(\Delta \alpha_{c} l_{c}\right)\right)^{2}}
$$

For the sign convention, the positive relation holds in the domain of $1<\exp \left(\Delta \alpha_{c} l_{c}\right)<1 / R_{T}$, while the negative relation holds when $\exp \left(\Delta \alpha_{c} l_{c}\right)>1 / R_{T}$. From (11), we can observe two features. The first one is that $\exp \left(\Delta \alpha_{c} l_{c}\right)$ increases with $\left|\Delta R_{\text {tot }}\right|$, i.e., as $\Delta \alpha_{c}$ increases, $\left|\Delta R_{\text {tot }}\right|$ increases. The second one is that, in the case of $\exp \left(\Delta \alpha_{c} l_{c}\right)>1 / R_{T}, \Delta \alpha_{c}$ and $\Delta R_{\text {tot }}$ are opposite in sign. For instance, if the operation state changes from OFF to ON, the change of absorption, i.e., $\alpha_{c}(F \neq 0$, OFF state $)-\alpha_{c}(F=0$, ON state $)$, becomes positive in sign, and the change of reflectance, i.e., $R_{\text {tot }}(\mathrm{OFF})-R_{\text {tot }}(\mathrm{ON})$, becomes negative. It should be noted that (11) can be used to estimate the effect of the change of absorption of the DFQW on the performance $\left(\Delta R_{\text {tot }}\right)$ of the modulator.

Another indicator of the effect of interdiffusion in the modulator performance is the absolute change of reflectance per change of photon wavelength, i.e., $|\Delta R / \Delta \lambda|$, around the dip of the operated ON-state FP mode. The wavelength of the FP mode shifts under bias due to $\Delta n_{c}$. The larger the $\Delta n_{c}$ caused by the applied field, the larger shifting of the photon wavelength at FP mode. The $R_{\text {tot }}$ is measured at the wavelength of the OFF-state FP mode while at this wavelength the ON-state $R_{\text {tot }}$ is obtained at its FP mode dropping edge. Therefore, a large $|\Delta R / \Delta \lambda|$ implies a large $\Delta R_{\text {tot }}$ at OFF state. The $|\Delta R / \Delta \lambda|$ can be represented by the finesse of the FP mode. The higher the finesse, the larger is the $|\Delta R / \Delta \lambda|$. The finesses can be calculated from the following relation:

$$
\text { Finesse }=\frac{\pi\left(R_{T} R_{B}\right)^{1 / 4} e^{-\frac{1}{2} \alpha_{c} l_{c}}}{1-\sqrt{R_{T} R_{B}} e^{-\alpha_{c} l_{c}}}
$$

taken from Whitehead et al. [33]. If $\Delta n_{c}$ increases, the FP mode under bias can shift further away from that of the ON state $(F=0)$, resulting in an increasing $\Delta R_{\text {tot. }}$. Therefore, $\Delta n_{c}$ of the DFQW also contributes to the variation of $\Delta R_{\text {tot }}$ in the DFQW FP modulator.

\section{RESUlts AND DisCUSSIONS}

In this paper, a $101-\AA / 100-\AA \mathrm{Al}_{0.3} \mathrm{Ga}_{0.7} \mathrm{As}-\mathrm{GaAs} \mathrm{DFQW}$ 's FP reflection modulator has been analyzed. The values of the DFQW material parameters to be used in the present model are taken as those in [15]. The top and bottom reflectors consist of the alternate $\mathrm{Al}_{0.3} \mathrm{Ga}_{0.7} \mathrm{As}$ and $\mathrm{AlAs}$ materials. The reflectance of the bottom reflector is set at a constant value of 0.97 . The top layer is adjusted, at OFF state, to vary and match the effective reflectance of the structure consists of both the absorptive cavity and the bottom reflector. It is assumed that there is no absorption loss resulting from both reflectors since the bandgap of the bulk alternative layers in the reflectors is too large to have any absorption at the selected operation wavelength. The refractive index of the materials in the Bragg reflectors are taken from [34] and [35]. The insertion loss of the DFQW modulator is considered to be caused mainly by the optical absorption in the active cavity. The total coupling efficiency is assumed to be unity. Since $\lambda_{\text {op }}$ will shift during interdiffusion of the cavity active material, the thickness of the mirror layers and their number of periods will be slightly re-adjusted in order to allow the modulator to operate at the impedance condition. Several measuring parameters, such as the change of reflectance, on-resonant reflectance at the OFFstate FP mode, operated voltage, relative reflectance change per unit voltage, chirping parameter, and adjustability of operation wavelength will be used to assess the performance of the modulator. Once the operation criteria of the modulator has been defined, optimum results can then be obtained. 


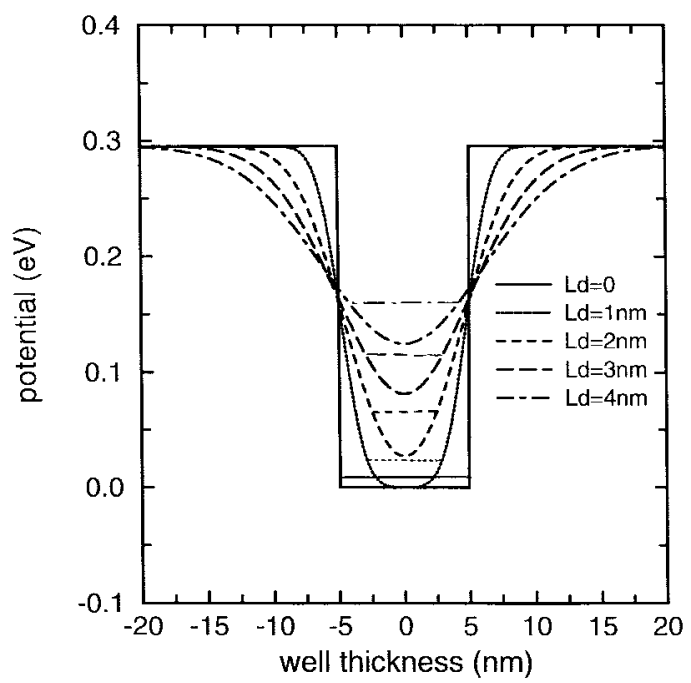

Fig. 3. Potential profile and the first energy state of DFQW's, $L_{d}=0$ (solid line), $L_{d}=10 \AA$ (dot line), $L_{d}=20 \AA$ (dash line), $L_{d}=30 \AA$ (long-dash line), and $L_{d}=40 \AA$ (dot-dash line).

\section{A. Outstanding Features of DFQW as the FP Modulator}

The characteristics of a good electroabsorptive QW FP modulator include low operation voltage, large $\Delta R_{\text {tot }} / V$, high $\Delta R_{\text {tot }}$, large $\Delta R_{\text {tot }} / R_{\text {tot }}(\min )$ or a high ON/OFF ratio, an adjustable operation wavelength, and small chirping parameter. In the following, we will explain how the DFQW, used as an active cavity material of the FP modulator, can contribute to these characteristics.

The conventional ways to achieve low voltage swing are: 1) to reduce the number of QW layers in the active cavity [5], or 2) in addition to 1), further modifies the composition of the aluminum in the AlGaAs-GaAs QW and at the same time reduce their periodic thickness [6]. Here, we propose the use of extensively diffused DFQW structures $\left(L_{d}=30 \AA\right.$ and $40 \AA$ ) as the active cavities for the FP modulators, to reduce the voltage swing. After an extensive interdiffusion $\left(L_{d}=\right.$ $40 \AA$ ), the potential barrier reduces in depth and becomes nonlinear in shape, as shown in Fig. 3. Thus, the carriers can have a higher tunneling rate [36] or equivalently, for a required ON/OFF ratio, a lower applied voltage as compared to that of the rectangular QW. This results in a larger $\Delta R_{\text {tot }} / V$.

The use of DFQW can also contribute to the figures of merit: high $\Delta R_{\text {tot }}$ and large $\Delta R_{\text {tot }} / R_{\text {tot }}(\min )$. Since the quantum confinement of the ground state is enhanced, the exciton absorption coefficient and the $\Delta \alpha_{c}$ increase in a slightly interdiffused $\mathrm{QW}$, in which $\Delta \alpha_{c}$ is measured at the biased exciton peak. The applied fields of $\mathrm{ON}$ and $\mathrm{OFF}$ states are shown in Table I. Referring to Fig. $4, \Delta \alpha_{c}$ of the DFQW improves over that of its as-grown rectangular QW for the cases between $L_{d}=10 \AA$ and $20 \AA$. This implies that, from (11), $\Delta R_{\text {tot }}$ of these DFQW's is larger than that of their as-grown rectangular QW. This feature alone can make the DFQW cavity structure to serve as a highreflectance-change FP modulator. In addition, the DFQW FP modulator structure can also achieve a very small value of $R_{\text {tot }}$ (min). Together with a high $\Delta R_{\text {tot }}$, the DFQW modulator can reach a high $\Delta R_{\text {tot }} / R_{\text {tot }}(\mathrm{min})$ ratio, which is important

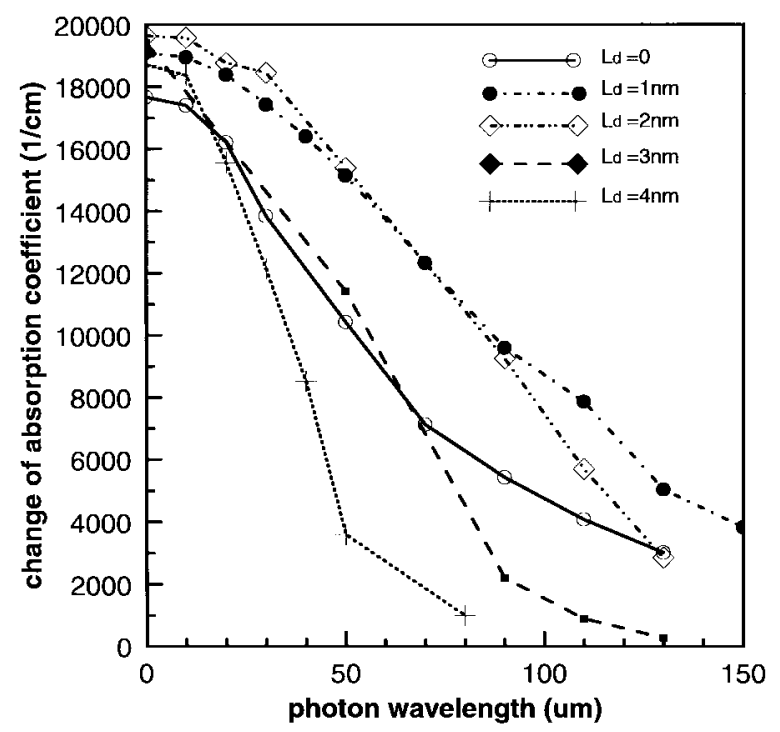

Fig. 4. The change of absorption coefficient of DFQW's $\left(L_{d}=0-40 \AA\right)$ in different OFF-state applied field. They are operated at exciton peak under different bias. The applied field of ON and OFF states are shown in Table I.

TABLE I

The Applied Field of ON ANd OFF States in the Operation of tHe Electroabsorptive DFQW FP REFLECTION MOdUlator

\begin{tabular}{lll}
\hline $\mathrm{L}_{\mathrm{d}} \AA$ & $\mathrm{F}(\mathrm{OFF}) \mathrm{kV} / \mathrm{cm}$ & $\mathrm{F}(\mathrm{ON}) \mathrm{kV} / \mathrm{cm}$ \\
0 & $0-60$ & 130 \\
0 & $70-130$ & 0 \\
10 & $0-90$ & 150 \\
10 & $110-150$ & 0 \\
20 & $0-110$ & 130 \\
20 & $120-130$ & 0 \\
30 & $0-110$ & 130 \\
30 & $120-130$ & 0 \\
40 & $0-60$ & 80 \\
40 & $70-80$ & 50 \\
\hline
\end{tabular}

in the development of switching networks for communication applications [37].

Another distinguished feature of the DFQW is its postgrowth adjustibility of $\lambda_{\mathrm{op}}$. During interdiffusion, the ground state of the DFQW rises as shown in Fig. 3. So the fundamental transition energy blue shifts and the photon wavelength reduces. In the traditional way, different rectangular QW's have to be fabricated on different substrate to produce modulators operating in different $\lambda_{\mathrm{op}}$. By using interdiffusion, we need only a single substrate QW structure with a selective interdiffusion process to develop a FP modulator which operates at a different photon wavelength. This can be used as an off-the-shelf technology to unify the as-grown QW material system and to improve the yield of fabrication.

To the electroabsorption FP modulator, the effect of chirping is also a serious parasite in modulation. Although DFQW cannot eliminate the chirping effect as it operates simultaneously with the electroabsorptive effect and the electrooptic effect, the chirping would not cause any adverse effect on our modulator. This is because its operation wavelength is far $(\geq 10 \mathrm{~nm})$ from the unbiased exciton absorption peak of the DFQW. In fact, the static chirping parameter of the DFQW structures has also been analyzed in a waveguide-type structure [38], [39]. 


\section{B. Operation Criteria of the DFQW FP Modulator}

Top and Bottom Bragg Reflectors In order to achieve a high-reflectance, high refractive-index change at the layered interface in the Bragg reflectors is necessary. Such change can be made by exposing one of the alternative layers of the top Bragg reflector with a higher refractive-index material to air. However, for the conventional $(H L)^{N}$ reflector structure [2], [5], [7], a one-period adjustment is too large to finetune the reflectance of this structure to the expected $R_{T}$. Alternatively, the $L(H L)^{N}$ structure, which provides a relatively gentle increment in reflectance, will be used here to modify the $R_{T}$ of the top reflector. Moreover, since $\lambda_{\mathrm{op}}$ of the DFQW modulator will shift, both the top and bottom Bragg reflectors have to be rearranged in order to obtain the expected modulation. The variation of $L_{d}\left(\Delta L_{d}=10 \AA\right)$ will induce significant changes to the optical properties of QW while maintaining a negligible effect on the thin-film (bulk material) Bragg reflector structure. The thickness of each of the Bragg reflector layers considered here is between 550 and $800 \AA$. Take the $550-\AA$ thickness as an example, the worst ( $L_{d}=40 \AA$ ) interdiffusion brings less than a $3-\mathrm{meV}$ variation in the fundamental transition energy and a $10-\AA$ variation of this $L_{d}$ only bring a maximum difference of $1 \mathrm{meV}$ in the transition energy of these layers. Hence, its effect on the optical parameters (such as reflectivity of the Bragg reflector) can be ignored.

Cavity Structure The condition for selecting a suitable $\lambda_{\mathrm{op}}$ is to set the OFF state at the exciton edge for large $\Delta \alpha$ while the ON state should be chosen for a low residual loss. However, there is a tradeoff between $\Delta \alpha$ and $\alpha_{\text {loss }}$. In order to minimize the dominant factor, i.e., the residence loss, $\lambda_{\mathrm{op}}$ is re-adjusted to $0.7713 \mu \mathrm{m}$, which is far from the exciton edge $(0.76 \mu \mathrm{m})$ under zero bias. This produces a small $\Delta \alpha$. On the other hand, the default structure of a 100$\AA / 100-\AA \mathrm{Al}_{0.3} \mathrm{Ga}_{0.7} \mathrm{As}-\mathrm{GaAs} \mathrm{DFQW}$ will be restructured to $101-\AA / 100-\AA \mathrm{Al}_{0.3} \mathrm{Ga}_{0.7} \mathrm{As}-\mathrm{GaAs}$, so that the total thickness of the active region will provide phase matching in the cavity. It should be noted that in designing our modulator, phase change in the cavity with thickness $l_{c}$ is selected as a multiple of $2 \pi$ for one round trip. This cavity condition is considered as a cavity phase-matching condition for the DFQW modulator. The DFQW modulator should satisfy this condition as well as the impedance condition. In order to make proper comparison, the cavity structure of an as-grown rectangular QW is very nearly the same as that of the DFQW FP modulator.

\section{Optimized Results}

As the QW's undergo interdiffusion, the $\lambda_{\mathrm{op}}$ shifts to a new wavelength, and the cavity phase-matching condition thus becomes invalid. The total phase change and total reflectance $R_{\text {tot }}$ (both under bias and no-bias) at this shifted $\lambda_{\text {op }}$ will no longer satisfy the phase and impedance conditions. As a consequence, the performance of the modulator varies as a function of the extent of interdiffusion. There are two ways to maintain the total phase-matching condition of the device. The first is to fix the cavity thickness $l_{c}$ for different cases of interdiffusion while rearranging the Bragg reflectors in order to compensate the phase-change deviation. It should be noted that not only the total number of periods of the reflector layers has to be adjusted, but also the thickness of each layer has to be altered for satisfying the quarter-wavelength condition. The second is to adjust the reflector parameters such as its number of periods and the thickness of each bulk layer. The $l_{c}$ should also be adjusted to achieve an $2 n \pi$ phase change in the DFQW in addition to the total phase-matching condition. Therefore, for different $L_{d}$ cases, the thickness of the device is adjusted in order to maintain the phase-matching condition. These two methods are now described as follows.

1) Fixed Cavity Design: The thickness of the DFQW cavity is fixed to be the same as that of the rectangular QW case $\left(l_{c}=1.2273 \mu \mathrm{m}\right)$. However, since the $\lambda_{\mathrm{op}}$ shifts while the QW structure undergoes interdiffusion, the top and bottom Bragg reflectors, the number of alternative layers, and the thickness of each quarter-wavelength layer have to be rearranged in order to match the predetermined $R_{T}$ and $R_{B}$ from the impedance condition set at the shifted $\lambda_{\mathrm{op}}$. The number of periods of the top and bottom reflectors are considered here to be 8.5 and 16.5 , respectively. If the as-grown rectangular wells $\left(L_{d}=0\right)$ are used in the cavity, the $\mathrm{AlAs}$ and $\mathrm{Al}_{0.3} \mathrm{Ga}_{0.7}$ As layers have thicknesses of 733 and $638 \AA$, respectively. When the QW structure undergoes an interdiffusion of $L_{d}=40 \AA$, the top and bottom reflectors will be redesigned to 7.5 and 16.5 periods of alternative AlAs (638 $\AA$ ) and $\mathrm{Al}_{0.3} \mathrm{Ga}_{0.7} \mathrm{As}(553 \AA)$ layers, respectively. Since $l_{c}$ of the DFQW is fixed at the same value as the rectangular QW, the total thickness of the device mainly depends on the thickness of the top and bottom reflectors. These reflectors will in turn depend on the extent of diffusion of the DFQW materials employed in the cavity in order to match the impedance condition.

Table II shows the modulation properties of the DFQW's modulators with identical thickness of cavity and the ON state of the device operating under a zero applied field. As can be seen, the $\Delta R_{\text {tot }} / R_{\text {tot }}(\min )$ performance of the modulator is unsatisfactory in various interdiffusion cases when the thickness is fixed in the cavity design. Although $\Delta R_{\text {tot }}$ in cases of $L_{d}=20$ and $30 \AA$ is kept above 0.5 , the $R_{\text {tot }}$ (min) of various $L_{d}$ 's is relatively high when compared to the rectangular QW $\left(L_{d}=0\right)$ case, in which the $R_{\text {tot }}(\min )$ is only $3.2924 \times 10^{-3}$. The high $R_{\text {tot }}(\min )$ can be explained by a shift of $\lambda_{\mathrm{op}}$ under interdiffusion, but the $l_{c}$ (tailor for operating in rectangular QW case) is now employed for operation at the shifted $\lambda_{\text {op }}$. Therefore, the cavity phase change is no longer a multiple of $2 \pi$. This means that the impedance condition cannot be fulfilled. Also, since phase change of the cavity varies with interdiffusion, the outermost layer of the top Bragg reflector has to be adjusted irregularly for different cases of $L_{d}$ to satisfy the quarter-wave condition. As a consequence, the simplified relation (7) may not hold and this results in poor modulation for most of the $L_{d}$ cases. In other words, it seems inappropriate to develop a "fixed cavity design" for the FP modulator.

We propose here a single-cavity structure to develop FP modulators with different $\Delta R_{\text {tot }}$ and finesse at similar $\lambda_{\text {op }}$ by setting impedance conditions at one of the different fields. The term "setting of impedance condition at a specific field" represents that the impedance condition for the modulator 
TABLE II

Features of the DFQW Reflection Modulators with an Identical Thickness of Cavity

\begin{tabular}{|c|c|c|c|c|c|c|c|}
\hline $\operatorname{Ld}(\AA)$ & $\lambda_{\mathrm{op}}(\mu \mathrm{m})$ & $\Delta R_{\text {lol }}$ & $\mathrm{R}_{\mathrm{tut}}(\min )$ & $\Delta \mathrm{R}_{\mathrm{tol}} / \mathrm{R}_{\mathrm{tot}}(\mathrm{min})$ & $\begin{array}{c}\mathrm{F}(\mathrm{kV} / \mathrm{cm}) \\
\text { [OFF- } \\
\text { STATE] }\end{array}$ & $\alpha_{\mathrm{loss}}\left(\mathrm{cm}^{-1}\right)$ & $\begin{array}{l}\text { device } \\
\text { thickness } \\
(\mu \mathrm{m})\end{array}$ \\
\hline 0 & 0.8731 & 0.6428 & $3.2924 \times 10^{-3}$ & 195.2 & 130 & 345 & 4.5180 \\
\hline 10 & 0.8564 & 0.4773 & 0.4339 & 1.10 & 130 & 569 & 3.5104 \\
\hline 20 & 0.8273 & 0.1805 & 0.7621 & 0.2368 & 130 & 601 & 3.5565 \\
\hline 30 & 0.7928 & 0.5438 & $5.262 \times 10^{-2}$ & 10.33 & 90 & 1063 & 4.3230 \\
\hline 40 & 0.7712 & 0.0402 & 0.9213 & 0.04368 & 50 & 667 & 3.4908 \\
\hline
\end{tabular}

TABLE III

Features of the DFQW Reflection Modulators with $l_{c}$ Nearest to that of the SQuare QW Modulator

\begin{tabular}{ccccccc}
\hline $\mathrm{Ld}(\hat{\mathrm{A}})$ & $\lambda_{\text {op }}(\mu \mathrm{m})$ & $\Delta \mathrm{R}_{\text {tot }}$ & $\mathrm{R}_{\text {tot }}(\min )$ & $\begin{array}{c}\mathrm{F}(\mathrm{kV} / \mathrm{cm}) \\
{[\mathrm{OFF}-\mathrm{STATE}]}\end{array}$ & $\mathrm{l}_{\mathrm{c}}(\mu \mathrm{m})$ & $\alpha_{\text {tose }}\left(\mathrm{cm}^{-1}\right)$ \\
\hline 0 & 0.8731 & 0.6428 & $3.2924 \times 10^{-3}$ & 130 & 1.2273 & 348 \\
10 & 0.8564 & 0.5722 & 0.1933 & 130 & 1.327 & 569 \\
$10(\mathrm{new})$ & 0.8607 & 0.7141 & $7.932 \times 10^{-6}$ & 150 & 1.217 & 333 \\
20 & 0.8270 & 0.6613 & $4.144 \times 10^{-3}$ & 130 & 1.282 & 601 \\
30 & 0.7991 & 0.5580 & $2.327 \times 10^{-4}$ & 110 & 1.240 & 461 \\
40 & 0.7713 & 0.6687 & $4.7633 \times 10^{-5}$ & 80 & 1.297 & 667 \\
\hline
\end{tabular}

TABLE IV

The Fractional Change of Reflectance Per Unit Square Voltage, Capacitance, and $f_{3} \mathrm{~dB}$ in Different $L_{d}$ 's Case with Tailored CaVity Thickness Considered in Table II

\begin{tabular}{cccccccc}
\hline Ld $(\AA)$ & $\Delta \mathrm{R}_{\mathrm{us}} / \mathrm{R}_{\text {wo }}(\mathrm{min})$ & $\begin{array}{c}\text { applied } \\
\text { voltage } \\
(\mathrm{V})\end{array}$ & $\begin{array}{c}\Delta \mathrm{R}_{\mathrm{to}} / \mathrm{V} \\
\left(\mathrm{V}^{-1}\right)\end{array}$ & $\begin{array}{c}\Delta \alpha_{\mathrm{c}} \\
\left(\mathrm{cm}^{-1}\right)\end{array}$ & $\Delta \mathrm{n}_{\mathrm{c}}$ & finesse & $\begin{array}{c}\text { phase change in } \\
\text { cavity (half-trip) }\end{array}$ \\
\hline 0 & 195.2 & -13.33 & 0.048 & 3013 & $-2.20 \times 10^{-2}$ & 2.47 & $10 \pi$ \\
$10(\mathrm{new})$ & 93457 & -14.48 & 0.049 & 3833 & $-1.88 \times 10^{-2}$ & 1.84 & $10 \pi$ \\
20 & 326.1 & -14.04 & 0.047 & 2860 & $-3.70 \times 10^{-2}$ & 2.29 & $11 \pi$ \\
30 & 159.2 & -10.94 & 0.051 & 900 & $-1.71 \times 10^{-2}$ & 6.52 & $11 \pi$ \\
40 & 14040 & -7.6 & 0.088 & 1007 & $-2.78 \times 10^{-2}$ & 5.29 & $12 \pi$ \\
\hline
\end{tabular}

structure is designed at a field under the OFF state. To facilitate our discussion, one of the cases, $L_{d}=0$, is shown in Fig. 5. By setting the impedance condition (OFF state) at $F=$ 0 (normally OFF operation) and $130 \mathrm{kV} / \mathrm{cm}$ (normally ON operation), good modulation performance can be obtained with their respective $\Delta R_{\text {tot }}=0.444$ and 0.643 , and $R_{\text {tot }}(\min )=$ $1.332 \times 10^{-3}$ and $3.292 \times 10^{-3}$. It should be noted that their ON states are operated under fields of $F=130 \mathrm{kV} / \mathrm{cm}$ and 0 respectively. The $\lambda_{\mathrm{op}}$ shifts from 0.8755 to $0.8730 \mu \mathrm{m}$ as a consequence of setting the bias impedance condition to the normally $\mathrm{ON}$ case. This is due to a change of the refractive index. As shown from Fig. 5(a), the normally OFF case can serve as a high finesse to provide a narrow-range FP modulator in order to filter out the noise from other photon wavelengths. A peculiar feature can also be observed that the $\mathrm{ON}$ and $\mathrm{OFF}$ reflectance spectra cross each other, thus giving rise to zero $\Delta R_{\text {tot }}$.

2) Tailored Cavity Design: The modulation can be further improved by adjusting the cavity thickness, $l_{c}$, for $2 n \pi$ phase variation under bias for different cases of $L_{d}$. The reflectance spectra for several applied fields are shown in Fig. 6(a) and (b) corresponding to cases of $L_{d}=0$ (rectangular $\mathrm{QW}$ ) and $L_{d}=40 \AA$, respectively. In the $L_{d}=0$ case, the OFF state (ON state) operates at $\lambda_{\mathrm{op}}=0.873 \mu \mathrm{m}$ with $F=130 \mathrm{kV} / \mathrm{cm}$ $(F=0)$. At this $\lambda_{\mathrm{op}}$, the reflectance are close to each other in cases of $F=0,50$, and $90 \mathrm{kV} / \mathrm{cm}$. This is because their absorption coefficients are merged together (see Fig. 7). Other characteristics of this FP modulator are shown in Tables III and IV.

In the case of $L_{d}=10 \AA, R_{\mathrm{tot}}(\mathrm{min})$ is too large to act as a high ON-OFF ratio modulator. The main reason is that the theoretical value of $R_{T}$ for the layered FP modulator, in which the $l_{c}$ of the DFQW cavity is selected as closest to that of the as-grown rectangular QW as possible, requires a value (0.0952 under impedance condition) which is too small (the best available value of $R_{T}$ is 0.0976 with a deviation of $2.5 \%$ ) to be fulfilled by the top layered Bragg reflector. This results in a deviation of $R_{\text {tot }}(\mathrm{min})$ from zero by 0.1933 and thus a reduction of $\Delta R_{\text {tot }}$ as compared to the $L_{d}=0$ case. Although $\Delta \alpha_{c}$ of the $L_{d}=10 \AA$ case at a field of $130 \mathrm{kV} / \mathrm{cm}$ is larger than that of the $L_{d}=0$ case (see Fig. 4), $\Delta R_{\text {tot }}$ reduces (not following the prediction from Section II-E) because $R_{\text {tot }}(\mathrm{min})$ is now too large to satisfy the proportional relation (11) of $\Delta R_{\text {tot }}$ and $\Delta \alpha_{c}$. It should be noted that the failure of this case is caused by the Bragg-reflector layered structure (which brings a large deviation of available $R_{T}$ from the predicted one) and not by the use of DFQW.

In order to solve the problem of failure in the Bragg reflector, a DFQW of $L_{d}=10 \AA$ with $\lambda_{\text {op }}=0.8607 \mu \mathrm{m}$ under a field of $150 \mathrm{kV} / \mathrm{cm}$ is used. In this case, the theoretical value of $R_{T}=0.2154$ can be achieved by designing the top reflector layer with a reflectance of 0.2181 (the deviation is only $0.5 \%$ ). 


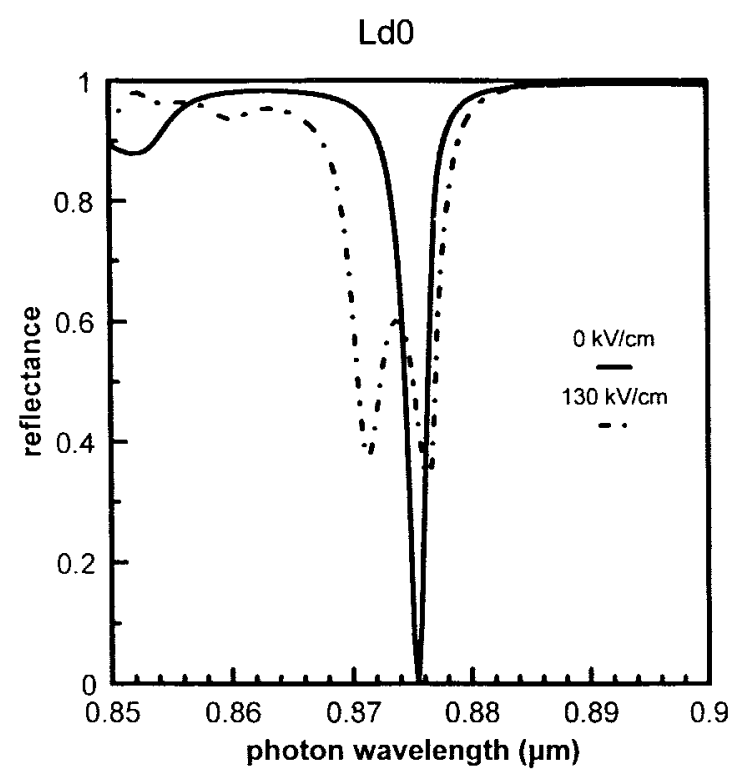

(a)

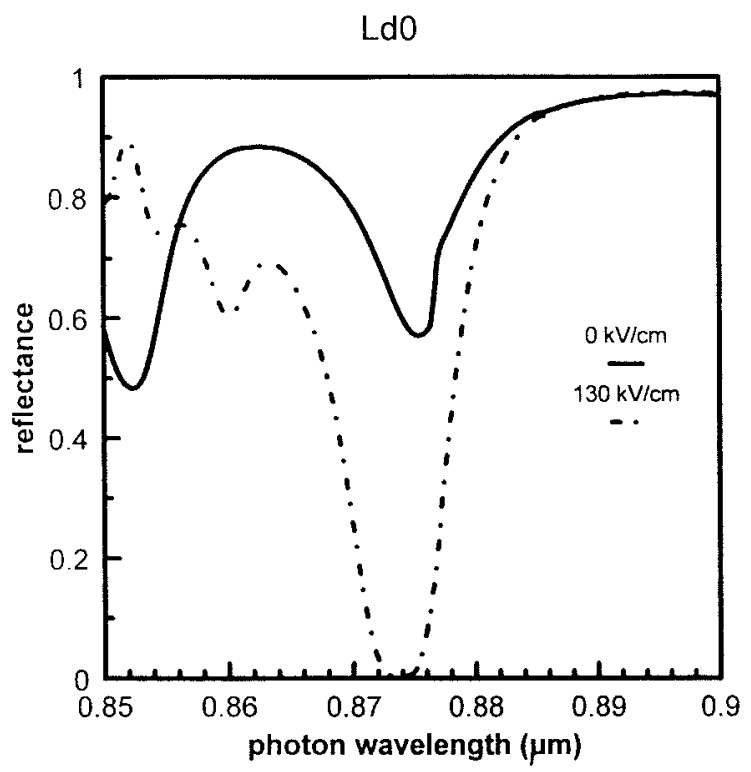

(b)

Fig. 5. The reflectance of the modulator $\left(L_{d}=0\right)$. The impedance condition is set in the situation the modulator under (a) $F=0 \mathrm{kV} / \mathrm{cm}$ (normally OFF operation), and (b) $F=130 \mathrm{kV} / \mathrm{cm}$ (normally $\mathrm{ON}$ operation).

This deviation is small enough to satisfy (11). The case, $L_{d}=$ $10 \AA$, can truly reflect the advantage of enlarging $\Delta \alpha_{c}$ through using DFQW in the FP modulator. This advantage results in an increase of $\Delta R_{\text {tot }}$ while, on the other hand, does not seriously reduce finesse and $\Delta n_{c}$. In the case of $L_{d}=20 \AA$, even though $\Delta \alpha_{c}$ and finesse are similar in magnitude with the case $L_{d}=$ 0 , its magnitude of $\Delta n_{c}$ is much enhanced. This means that the FP mode under bias has a larger shift from the ON state and results in an improved $\Delta R_{\text {tot }}$.

One of the other contributions of DFQW to FP modulator is the improvement of finesse in the more extensively interdiffused QW ( $L_{d}=30$ to $40 \AA$ ) which results in an enhancement of the $\Delta R_{\text {tot }}$. In the $L_{d}=30 \AA$ case, when compared to the $L_{d}=0$ case, although $\Delta \alpha_{c}$ reduces from 3013 to $1007 \mathrm{~cm}^{-1}$,

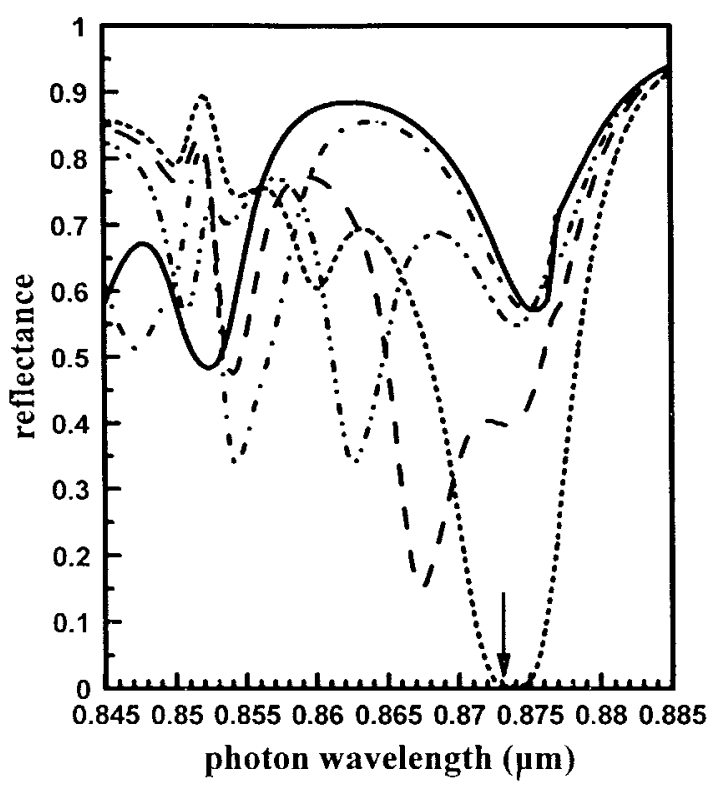

(a)

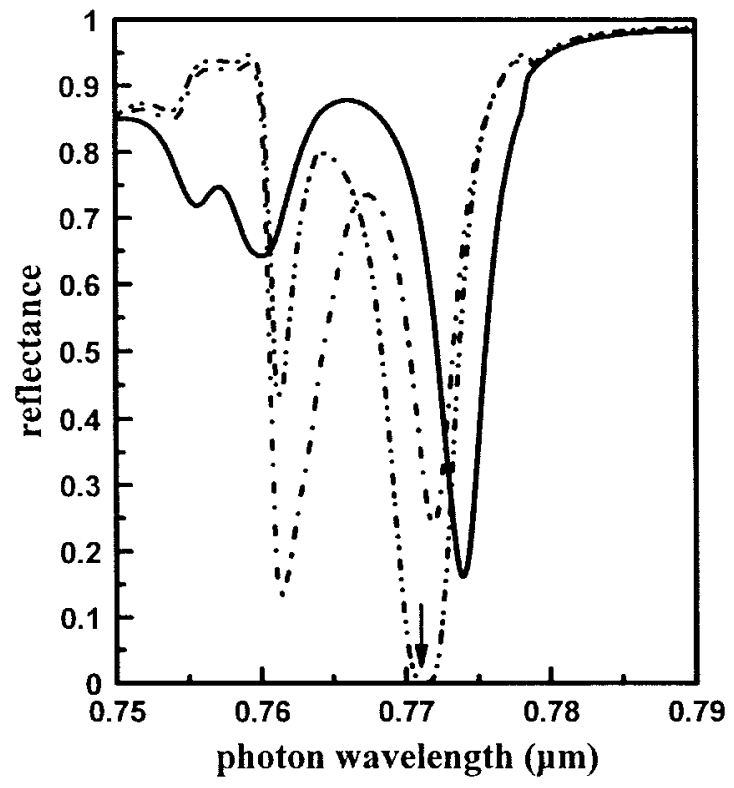

(b)

Fig. 6. (a) The reflectance spectra of $L_{d}=0$ with field $F=0$ (solid), 50 (dash-dot), 90 (dash-double dot) and 110 (long dash) and $130 \mathrm{kV} / \mathrm{cm}$ (dot). (b) The reflectance spectra of $L_{d}=40 \AA$ with field $F=0$ (solid), 50 (dash-dot), $80 \mathrm{kV} / \mathrm{cm}$ (dash-double dot). The arrows shown in the figures indicate the position of operation wavelength.

causing a small rise in the on-resonant $R_{\text {tot }}$ of the FP mode from the OFF to the ON state and $\Delta n_{c}$ also reduces by $23 \%$, the finesse increases about threefold. As a consequence, the value of $\Delta R_{\text {tot }}$ only slightly reduces to 0.5580 as compared to that of $L_{d}=0$, and, which is large enough to serve as a high-reflectance-change modulator [6].

As the interdiffusion further proceeds to $L_{d}=40 \AA$, the finesse still increases more than twofold while $\Delta n_{c}$ increases by a factor of 1.25 compared with its as-grown rectangular QW case. On top of these, $\Delta \alpha_{c}$ also improves as compared to the case of $L_{d}=30 \AA$. The $\Delta R_{\text {tot }}$ thus increases to 0.6687 . In addition, another excellent attribute of the application of 


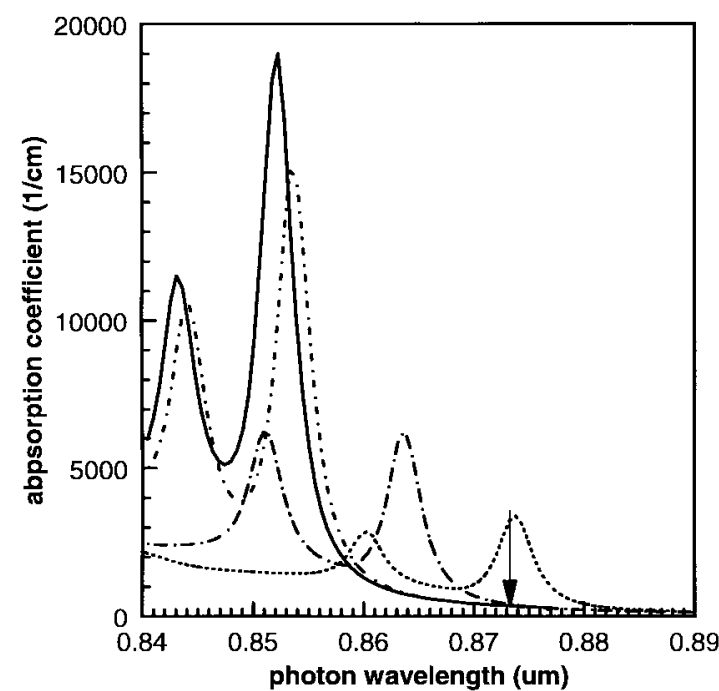

Fig. 7. The absorption spectra $(1 / \mathrm{cm})$ of $L_{d}=0$ with applied field $F=0$ (solid), 30 (dash-dot), 50 (dash-double dot), $90 \mathrm{kV} / \mathrm{cm}$ (long dash), and 130 $\mathrm{dV} / \mathrm{cm}$ (dotted line).

DFQW ( $\left.L_{d}=40 \AA\right)$ in the modulator is that only half of the applied voltage as that in $L_{d}=0$ is required in the operation. This figure of merit together with the high reflectance change per voltage form the best DFQW case (see Table IV). Furthermore, the operation wavelength can be adjusted by interdiffusion from $0.8733 \mu \mathrm{m}\left(L_{d}=0\right)$ to $0.7713 \mu \mathrm{m}\left(L_{d}=40 \AA\right)$ with a tunable range of more than $100 \mathrm{~nm}$. However, the tolerance of this $\left(L_{d}=40 \AA\right)$ DFQW FP modulator, which is analyzed by the FWHM of its contrast ratio $(\mathrm{CR})$ spectrum, is only $1.1 \mathrm{~nm}$. Although the tolerance is small, the drawback can be completely solved by the interdiffusion technology proposed here, i.e., by finely and precisely tuning the operation wavelengths of the device to the expected value. For instance, a lower interdiffusion rate at 900 ${ }^{\circ} \mathrm{C}$ annealing temperature [40], [41] can be used to precisely control the extent of interdiffusion for modifying the operation wavelength without serious reduction in both finesse and $\Delta n_{c}$. From our results, the CR at $\lambda_{\mathrm{op}}=0.7713 \mu \mathrm{m}$ is as high as 41 $\mathrm{dB}$. This makes the DFQW cavity an attractive structure for developing high-performance FP modulators.

According to the figure of merit for high $\Delta R_{\mathrm{tot}} / R_{\mathrm{tot}}$ (min), the $R_{\text {tot }}(\mathrm{min})$ of the DFQW FP modulator is determined to be approximately zero as expected from (7). However, during fabrication, the nonuniformity of the stacked structure makes the $R_{\text {tot }}(\mathrm{min})$ deviate further away from the absolute zero. Although the theoretical value of $R_{\text {tot }}(\min )$ in the DFQW, which we present here, is smaller than its as-grown rectangular $\mathrm{QW}$, it is expected that the realistic $\Delta R_{\text {tot }} / R_{\text {tot }}(\mathrm{min})$ value of the DFQW modulator should degrade when being fabricated. Nonetheless, $\Delta R_{\text {tot }}$ of the DFQW in some $L_{d}$ cases are larger than that of its as-grown rectangular $\mathrm{QW}$, according to the predicted value as shown in Table III. Thus, the DFQW modulator would be an interesting candidate in developing a high-performance modulator. With regard to chirping of the modulator, $\beta$, which is calculated from the relation $\beta=$ $\Delta n / \Delta k$ (where $\Delta k$ is the change in extinction ratio), its magnitude is ranging from $-1.39 \times 10^{-4}$ to $-4.98 \times 10^{-6}$ for the five $L_{d}$ cases. This means that chirping is not a significant problem for this type of DFQW electroabsorptive FP modulator considered here. Details of the static chirping parameter of DFQW can be found in [38].

\section{CONCLUSION}

We have presented a comprehensive analysis and theoretical optimization of the $101-\AA / 100-\AA \mathrm{Al}_{0.3} \mathrm{Ga}_{0.7} \mathrm{As}-\mathrm{GaAs} \mathrm{DFQW}$ structure in the asymmetric electroabsorptive FP reflection modulator. The biased voltage is also considered by applying Poisson's equation in the layered Bragg reflector structure with different dielectric constants. It is observed that the DFQW provides three advantageous features which can improve the performance of the FP modulator. They are as follows.

1) $\Delta \alpha_{c}$ of the DFQW (with $L_{d} \leq 20 \AA$ ), under different applied fields, generally increases over that of the $L_{d}=$ 0 case (rectangle $\mathrm{QW}$ ). Since $\Delta R_{\text {tot }}$ increases with $\Delta \alpha_{c}$, the modulation performance of the DFQW device improves.

2) In cases of more extensive interdiffusion, such as $L_{d} \geq$ $30 \AA$, electrons have a higher tunneling rate under bias, which implies that a smaller applied voltage is required.

3) As the fundamental transition energy increases with interdiffusion, the operation wavelength can therefore be tuned accordingly. Basing on this, we propose DFQW FP-modulator structures for different $L_{d}$, s which are modified from a rectangular QW FP modulator with a higher modulation performance.

The improvement of $\Delta n_{c}$ and finesse, and the features 1)-3) mentioned above, all contribute to the enhancement of $\Delta R_{\text {tot }}$. In addition, an increasing $\Delta R_{\text {tot }} / V$ ratio also results as the interdiffusion becomes extensive. An adjustable operation wavelength of over $100 \mathrm{~nm}$ ranges from $0.8833 \mu \mathrm{m}\left(L_{d}=0\right)$ to $0.7713 \mu \mathrm{m}\left(L_{d}=40 \AA\right)$, can also be achieved. Moreover, the OFF-state on-resonance reflectance is almost close to zero. Finally, with such a simple annealing process to produce the DFQW FP modulator, there is no doubt that it will become a very competitive candidate among the reflection type of modulators. Future work, such as the redesigning of well width, material composition, and the consideration of special interdiffusion process, still needs to be done in order to optimize the modulator performance.

\section{REFERENCES}

[1] M. Whitehead, G. Parry, and P. Wheatly, "Investigation of etalon effects in GaAs-AlGaAs multiple quantum well modulators," Proc. Inst. Elect. Eng., vol. 136, Pt. J, pp. 52-58, 1989.

[2] R. H. Yan, R. J. Simes, and L. A. Coldren, "Analysis and design of surface-normal Fabry-Perot electrooptic modulators," IEEE J. Quantum Electron., vol. 125, pp. 2272-2280, 1989.

[3] P. Zouganeneli, P. J. Stevens, D. Atkinson, and G. Parry, "Design trade-offs and evaluation of the performance attainable by GaAs- $\mathrm{Al}_{0.3} \mathrm{Ga}_{0.7} \mathrm{As}$ asymmetric Fabry-Perot modulators," IEEE J. Quantum Electron., vol. 31, pp. 927-943, 1995.

[4] A. Tomita, Y. Kohga, and A. Suzuki, "5: 1 on-off contract InGaAs/InP multiple quantum well fabry-perot etalon modulator," Appl. Phys, Lett., vol. 55, pp. 1817-1819, 1989.

[5] R. H. Yan, R. J. Simes, and L. A. Coldren, "Surface-normal electroabsorption reflection modulators using asymmetric Fabry-Perot structures," IEEE J. Quantum Electron., vol. 27, pp. 1922-1931, 1991. 
[6] C. H. Lin, J. M. Messe, and Y. C. Chang, "A low-voltage highreflectance-change normally off refractive $\mathrm{GaAs} / \mathrm{Al}_{0.2} \mathrm{Ga}_{0.8} \mathrm{As} \mathrm{MQW}$ reflection modulator," IEEE J. Quantum Electron., vol. 30, pp. 1234-1240, 1994.

[7] K. K. Law, J. L. Merg, and L. A. Coldren, "Supperlattice surface-normal asymmetric Fabry-Perot reflection modulators: Optical modulator and switching," IEEE J. Quantum Electron., vol. 29, pp. 727-739, 1993.

[8] B. L. Weiss, Ed., Opt. Quantum Electron., Special Issue, vol. 23, pp. S799-S994, 1991.

[9] J. Bleuse, G. Bastrad, and P. Voisin, "Electric-field-induced localization and oscillatory electro-optical properties of semiconductor superlattices," Phys. Rev. Lett., vol. 60, pp. 220-230, 1988.

[10] B. Joseph, K. W. Goossen, J. M. Kuo, P. F. Kopf, D. A. B. Miller, and D. S. Chemla, "Room-temperature electroabsorption and switching in a GaAs/AlGaAs supperlattice," Appl. Phys. Lett., vol. 55, pp. 340-342, 1989.

[11] J. Fritz, T. M. Breman, B. E. Hammons, A. J. Harward, W. Worobey, G. A. Vanter, and D. R. Myers, "Low-voltage vertical-cavity transmission modulator for $1.06 \mu \mathrm{m}$," Appl. Phys. Lett., vol. 63, pp. 494-496, 1993.

[12] E. E. Mindezm, F. A. Rueda, and J. M. Hong, "Stark localization GaAsGaAlAs superlattices under an electric field," Phys, Rev. Lett., vol. 60 , pp. 220-223, 1988.

[13] M. Ghisoni, G. Parry, M. Pate, G. Hill, and J. Roberts, "Post growth fabrication of $\mathrm{GaAs} / \mathrm{AlGaAs}$ reflection modulators via impurity free disordering," Jpn. J. Appl. Phys., vol. 30, pp. L1018-L1020, 1991

[14] E. H. Li and B. L. Weiss, "Analytical solution of the subbands and absorption coefficients of AlGaAs-GaAs hyperbolic quantum wells," IEEE J. Quantum Electron., vol. 29, pp. 331-321, 1990.

[15] E. H. Li, B. L. Weiss, and K. S. Chan, "Effect of interdiffusion on the subbands in an $\mathrm{Al}_{x} \mathrm{Ga}_{1-x} \mathrm{As} / \mathrm{GaAs}$ single-quantum-well structure," Phys. Rev. B, vol. 46, pp. 15181-15192, 1992.

[16] J. Cibert, P. M. Petroff, D. J. Werder, S. J. Pearton, A. C. Gossard, and J. H. English, "Kinetics of implantation enhanced interdiffusion of Ga and Al at GaAs-GaAlAs interfaces," Appl. Phys. Lett., vol. 49, pp. 223-225, 1986.

[17] T. E. Schlesinger and T. Kuech, "Determination of the interdiffusion of $\mathrm{Al}$ and $\mathrm{Ga}$ in $(\mathrm{Al}, \mathrm{Ga}) \mathrm{As} / \mathrm{GaAs}$ quantum well," Appl. Phys. Lett., vol. 49, pp. 519-521, 1986

[18] J. D. Ralston, W. J. Schaff, D. P. Bour, and L. F. Eastman, "Room-temperature exciton electroabsorption in partially intermixed GaAs/AlGaAs quantum well waveguides," Appl. Phys. Lett., vol. 54, pp. 534-536, 1989.

[19] P. L. Thornton, W. J. Morsby, and H. F. Chung, "Unified planar process for fabricating heterojunction bipolar transistors and buriedheterostructure lasers utilizing impurity-induced disordering," Appl. Phys. Lett., vol. 53, pp. 2669-2671, 1988.

[20] W. X. Zou, J. L. Mers, R. J. Fu, and C. S. Hong, "Very-low-threhold, strained $\operatorname{In}_{x} \mathrm{Ga}_{1-y} \mathrm{As} / \mathrm{GaAs}$ quantum well lasers defined by impurityinduced disordering," IEEE Photon. Technol. Lett., vol. 3, pp. 400-402, 1991.

[21] E. Kapon, N. G. Stoffel, E. A. Dobisz, and R. Bhat, "Birefringent channel waveguides defined by impurity-induced superlattice disordering," Appl. Phys. Lett., vol. 52, pp. 351-353, 1988.

[22] S. R. Andrew, J. A. March, M. C. Holland, and A. H. Kean, "Quantumwell laser with integrated passive waveguides fabricated by neutral impurity disordering," IEEE Photon. Technol. Lett., vol. 4, pp. 426-428, 1992.

[23] D. A. B. Miller, J. S. Weiner, and D. S. Chemla, "Electric-field dependence of linear optical properties in quantum well structures: Waveguides electroabsorption and sum rules," IEEE J. Quantum Electron., vol. QE-22, pp. 1816-1830, 1986.

[24] J. D. Ralston, S. O'brien, G. W. Wicks, and L. F. Eadtman, "Roomtemperature exciton transitions in partially intermixed GaAs/AlGaAs superlattices," Appl. Phys. Lett., vol. 54, pp. 1511-1513, 1988.
[25] T. E. Schlesinger and T. Kuech, "Determination of the interdiffusion of $\mathrm{Al}$ and $\mathrm{Ga}$ in undoped (Al,Ga) As/GaAs quantum well," Appl. Phys. Lett., vol. 49, pp. 519-521, 1986.

[26] J. C. Lee, T. E. Schlesinger, and T. Kuech, "Interdiffusion of Al and Ga in (Al,Ga) As/GaAs superlattice," J. Vac. Sci. Technol. B, Microelectron. Process. Phenom., vol. 5, pp. 1187-1190, 1987.

[27] K. Kash, B. Tell, P. Grabbe, E. A. Dobisz, H. G. Graighead, and C. M. Tamargo, "Aluminum ion-implantation enhanced intermixing of GaAsAlGaAs quantum-well structures," J. Appl. Phys., vol. 63, pp. 190-194, 1988.

[28] Y. Hirayama, "Mechanism of Ga implantation enhances interdiffusion of $\mathrm{Ga}$ and $\mathrm{Al}$ at GaAs-GaAlAs interfaces," Appl. Phys. Lett., vol. 49, pp. 223-225, 1986.

[29] E. H. Li, B. L. Weiss, K. S. Chan, and J. Micallef, "Polarization dependent refractive index of an interdiffusion induces $\mathrm{AlGaAs} / \mathrm{GaAs}$ quantum well," Appl. Phys. Lett., vol. 62, pp. 550-552, 1993.

[30] F. Bassani and G. P. Parravicini, Electronic States and Optical Transitions in Solids. Oxford, U.K.: Pergamon, 1975, ch. 5.

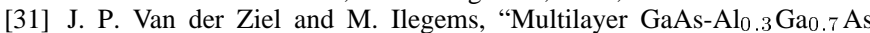
dielectric quarter wave stacks grown by molecular bean epitaxy," Appl. Opt., vol. 14, pp. 2627-2630, 1975.

[32] K. K. Law, L. A. Coldren, and J. L. Merz, "Low-voltage superlattice asymmetric fabry-perot reflection modulator," IEEE Photon. Technol. Lett., vol. 3, pp. 324-326, 1991.

[33] M. Whitehead, A. Rivers, and G. Parry, "Low-voltage multiple quantum well reflection modulator with on-off ratio > 100:1," Electron. Lett., vol. 25, pp. 984-985, 1989.

[34] B. Jensen and A. Torabi, "Dispersive of the refractive index of GaAs and $\mathrm{Al}_{x} \mathrm{Ga}_{1-x}$ As," IEEE J. Quantum Electron., vol. QE-19, pp. 877-882, 1983.

[35] R. E. Fern and A. Onton, "Refractive index of AlAs," J. Appl. Phys., vol. 42, pp. 3499-3500, 1971

[36] Z. Yang, B. L. Weiss, and E. H. Li, "The eigenstates and tunneling lifetime in an interdiffused $\mathrm{AlGaAs} / \mathrm{GaAs}$ quantum well by the transfer matrix method," Superlattice Microstruct, vol. 17, no. 2, pp. 177-186, 1995.

[37] J. E. Midwinter, "Novel approach to the design of optically activated wide band switching matrices," Proc. Inst. Elect. Eng., vol. 134, pp. 261-268, 1987

[38] E. H. Li and W. C. H. Choy, "Electro-optic and electro-absorptive modulation properties in interdiffusion modified $\mathrm{AlGaAs} / \mathrm{GaAs}$ quantum wells," IEEE Photon. Technol. Lett., vol. 7, pp. 881-883, 1995.

[39] W. C. H. Choy and E. H. Li, "Electro-absorptive and optic diffusedquantum-well modulators," in Proc. IOOC'95, 1995, pp. 142-144.

[40] P. J. Hughes, E. H. Li, and B. L. Weiss, "Thermal stability of AlGaAs/GaAs single QW structures using photo reflectance," J. Vac. Sci. Technol. B, Microelectron. Process. Phenom., vol. 13, pp. 2276-2280, 1995.

[41] B. L. Weiss, I. V. Bradeley, N. J. Whitehead, and J. S. Roberts, "Disordering of AlGaAs/GaAs quantum well structures using low doses oxygen implantation," J. Appl. Phys., vol. 71, pp. 5715-5719, 1992.

Wallace C. H. Choy (S'96), photograph and biography not available at the time of publication.

E. Herbert Li (S'87-M'88-SM'95), photograph and biography not available at the time of publication. 